\title{
Immune Response and Pathogenesis of COVID-19 and The Strategies for Developing Target Drugs
}

\section{Anju Kaushal*}

Assistant Professor (Former), Shiva Group of Institutions Bilaspur, HP, India

*Corresponding Author: Anju Kaushal, Auckland, New Zealand.
Received: August 03, 2020

Published: August 26, 2020

(C) All rights are reserved by Anju Kaushal.

\section{Abstract}

Current pandemic with COVID-19 disease is caused by a novel coronavirus SARSCoV-2, which started from Dec 2019 and still continues. The infection is mainly started by inhalation of virus contaminated droplets. Immune response is produced by both adaptive and innate immunity. Pathogenic stages of virus in the host are asymptomatic stage, non-severe symptomatic and severe- symptomatic stage. Major- Histocompatibility Antigens/HLA classes regulate the adaptive immune response and pathogenesis. In asymptomatic stage the person is recovered normally. However, the innate immune response is impaired in the severe symptomatic stages with high lymphopenia in natural killer cells, but other macrophages and monocytes increased fighting the virus; hence they establish a hyperinflammatory state. Immunological regular functions are precluded mainly due to impaired immune response, because virus lapse the protective immunity by NK cells, while favoring its propagation causing inflammations in lungs and other organs such as kidney, liver, spleen etc., could be fatal in later stages.

ACE-2 receptors on alveolar cells with proteaseTMPRSS2 allow the spike of virus to make them attach to the cells for easy virus entry. Monocytes and Macrophages produce cytokines can create "Cytokine storm", a hyper inflammation in lungs. Cytokine storm is a current hallmark of SARSCoV-2 pathogenesis, is triggered by releasing cytokines GSCF, IP10, MIP1A, IL-2, IL-7 and TNF to cause breathing problem, ARDS, and lungs failure subsequently with acute cardiac injury.

Evidence based medicines to integrate the clinical experience and patient values with the accurate information available, would provide best treatment for faster patient recovery.

Keywords: COVID-19; SARSCoV-2; ACE-2; ARDS; Immune Response; Cytokines

\section{Abbreviations}

ACE-2: Angiotensin Converting Enzymes-2; APC: Antigen Presenting Cell; ARDS: Acute Respiratory Distress Syndrome; AP-1: Transcription Factor; ARB: Angiotensin Receptor Blocker; BALF: Bronchoalveolar Lavage Fluid; COVID-19: coronavirus infectious disease; CCL2: Chemokine Ligand 2; CCL3: Chemokine Ligand 3; CCL5: Chemokine Ligand 5; CXCL8: Chemokine (C-X-C motif) Ligand 8; CXCL10: Chemokine (C-X-C motif) Ligand 10; CD40L: CD 40 Ligand; CXCR3: Chemokine (C-X-C) motif Ligand 13; CD8 CELLS:
Cluster of Differentiation 8 cells; CD4 CELLS: Cluster of Differentiation 4 Cells; CRS: Cytokine Release Syndrome; DIC: Disseminated Intravascular Coagulation; D-Dimer: Fibrin Degradation Product; GSCF: Granulocyte Colony Stimulating Factor; HLA: Class Human Leukocyte Antigen Class; HLH: Hemophagocytic-lymphohistiocytosis; HA: Hyaluronan; HAS2: Hyaluronan Synthase; hsCRP: High Sensitivity C-reactive Protein; IP10: Induced Protein (or Small Inducible Cytokine); IL-2: Interleukin -2; IL-7: Interleukin-7; IRF7: Interferon Regulatory Factor 7; IFN1: Interferon; ICs: Intensive 
Care; IL1ß: Interleukin 1 Beta; IL6: Interleukin 6; IRF3: Interferon Regulatory Factor 3; IL-13: Interleukin 13; ICU: Intensive Care Unit; IFN: Stimulated Genes ISGs; IFN $\gamma$ : Interferon gamma; IL-17A: Interleukin 17 A; IVIG: Intravascular immunoglobulin; LMWH: Low Molecular Weight Heparin; MSCs: Mesenchymal Stromal/ Stem Cells; MCP1: Monocyte Chemoattractant Protein 1; 4MU: Methylumbelliferon; MHC: Major Histocompatibility; MyD88: Myeloid Differentiation Factor 88; MIP1A: Macrophage Inflammatory protein 1 Alpha; mRNA: Messenger Ribonucleic Acid; NK CELLS: Natural Killer Cells; Nabs: Neutralizing Antibodies; P38MAPK: p38 Mitogen Activated Protein Kinases; RBD RECEPTORS: Receptor Binding Domain; S-PROTEIN: Spike Protein of Virus; SARS CoV2: Severe Acute Respiratory Syndrome Coronavirus-2; TLR 7/8, 3: Toll like receptor 7/8; TH1- nor TC1- Helper and Cytotoxic Tcell subsets; TH2 - T helper type 2; TNF: Tumor Necrosis Factor; TRAF6: TNF Receptor Associated Factor 6 (TNF-Tumor Necrosis Factor); TLRs: Toll Like Receptors; TMPRSS2: Transmembrane Protease Serine 2; TLR 8: Toll- Like Receptors 8; TLR 7: Toll - Like Receptors; TNF $\alpha$ : Tumor Necrosis Factor Alpha; T/B CELLS: Thymus/Bone marrow or Bursa Derived Cells.

\section{Introduction}

The pandemic of disease COVID-19 is ongoing at present, and caused by a novel virus severe acute respiratory syndrome -2 (SARS-CoV-2). As of Aug 2020, more than 18 million cases of COVID-19 have been reported in more than 188 countries and territories, resulting in more than 680,000 deaths; and more than 11 million people have recovered. The virus is mainly spread among people during close contact, often via contaminated droplets produced by infected person while coughing, sneezing and talking. People may become infected by touching the contaminated surfaces too. The virus is more contagious during the first three days after the onset of symptoms; however, the spread is possible even before the symptoms appear. Common symptoms are fever, cough, fatigue, shortness of breath and loss of smelling sense.

The immune response and pathogenesis are produced in two phases: asymptomatic (Non -Severe stage) and symptomatic (Severe stage). In non - severe the stage the protective immunity is regulated by general good health and MHC-Antigens, which could lead to autoimmune disease. If the protective immunity is impaired, it allows the virus to disseminate to the various organs and can cause organ failure especially in lungs, heart, kidney, liver and others.
There is a heterogeneity observed in immune responses. In case of SARSCoV-2 infected individuals, lymphopenia is reported with deficiency of Natural Killer NK- cells. In asymptomatic individuals, the protective immune response is generated in a regular manner to clear the virus. However, in symptomatic individuals, the immunity is impaired to let the virus escape the first two protective immune pathways recognized by TLRs viz. MyD88 and IRF7 to produce IFN-1 and the TRAF6 and IRF3 to activate T-cells. However, it activates the third pathway through p38MAPK and AP-1 to hyperproduce the pro-inflammatory molecules. These pro-inflammatory conditions reported in patients in ICs, to endeavor monocytes and macrophages to synthesize chemokines (CCL2, CCL3, CCL5, CXCL8, CXCL10) and cytokines (IL-1 $\beta$, IL-6, TNF- $\alpha$ ). They help in disease progression with depositing the cells in various tissues, causing hyper-inflammation especially in lungs result into ARDS and consequently progressing towards lungs failure.

The drugs are being tested for repurposing to treat COVID-19 patients. These are: i) the antivirals which can control the virus cycle; and ii) others are anti-inflammatory can control the development of symptoms leading to disease severity.

This review article is providing the recommendations for further studies/and investigations to develop the target drugs to control the various pathogenic outcomes before even they develop. The diagnostics could also be developed to detect the pro-inflammatory molecules in the early stages, in order to begin the treatment right on time to control the organ damage and subsequent fatalities

An outlook on the immune response of COVID-19 disease

COVID-19 disease is an emerging viral threat with major repercussions for public health. There is not specific treatment for COVID-19 yet. The virus transmits from person to person at a close distance via infectious droplets. All people exposed to SARS CoV-2 don't get infected and not all infected individuals develop severe respiratory illness. Scientists and clinicians have recognized its pathogenesis in three stages:

- $\quad$ Stage 1- Asymptomatic incubation period with and without detectable virus.

- $\quad$ Stage 2- Non-severe symptomatic period with presence of virus.

- $\quad$ Stage 3- Severe respiratory symptomatic stage with high viral load. 
Persons in asymptomatic state are able to spread the virus unknowingly and they are least manageable. First asymptomatic case was reported in Germany [31,36,38]. Their role in spreading the infection is still undefined.

Elderly patients over 65 years of age could easily progress to the severe stage. Severity of the disease is not always depending upon the overall immunity of a person.

During the incubation and non-severe stage, an immediate adaptive immune response is needed to eliminate and deter the disease progression in to severe stages. Hence, it is imperative to boost immune response strategically at this stage by anti-sera or pegylated IFN $\alpha$.

Immune response is mainly generated due to good health and MHC-Antigens/HLA classes, could lead to autoimmune disease later, in the non-severe stage. Genetic differences also contribute to the different immune responses at an individual level. When a protective immunity is impaired, virus will disseminate to various organs of the body causing massive destruction, especially in lungs, intestine and kidney, are also known to have more ACE receptors. Innate inflammation in lungs is largely mediated by pro-inflammatory macrophages and granulocytes, leads to severe stage causing a life-threatening respiratory disorder [38]. Once the severe lung damage occur efforts should be made to suppress the inflammation in managing the severity of symptoms.

Some patients after discharge from hospital, again come back due to disease exacerbation, which is considering to the minimum immune response and virus lapse. This shows that vaccines would not work in those individuals. The recovered patients from nonsevere stage should be monitored for T/B cell responses. A crucial point for vaccine development and control testing.

\section{Dilemma in immunogenic interactions}

The adaptive and innate immune responses are involved with its cellular and soluble components. For cytopathic viruses, like influenza, the early phase immune intervention involves interferon (IFN-1), natural Killer cells (NK cells) to generate cytolytic CD8+ Tcells, able to control viremia in 3-4 days. The first low affinity IgM Abs appear in 6-7 days followed by IgG, IgA Abs to be developed in 3-4 weeks' time. Abs don't involve in recovery process, but are to be used for secondary infection and in seroprophylaxis [30].
In severe stage of SARS CoV-2, lymphopenia is reported by the deficiency of NK cells. The studies showed that in asymptomatic individuals, the innate response could control the virus and detection of CD8+ T-cells in peripheral blood provides the clue of cell mediated immunity. But, in case of symptomatic COVID-19, the Tcell mediated response escape the detection. Some data is available on the recruitment of NK cells and T-cell subsets and their functions (scRNAseq) in the broncheoalveolar lavage fluid of patients with pneumonia $[25,26]$. More information on the innate immune response is required for further justification.

There is a limited information about specific and neutralizing antibodies (Nabs), crucial for protective immunity. Antibodies against specifically RBD receptor-binding domain are considered as the NAbs neutralizing antibodies in coronavirus. Hyperimmune globulins from recovered patients may allow successful seroprophylaxis. However, some Nabs also target the non-RBD regions of S -protein would lead to cause antibody-dependent enhancement effect to exacerbate the disease. In a study, the recovered 26 patients had developed NAbs, but only 3 were showing specificity for RBD $[8,30]$. Though, this has raised a significant concern regarding the establishment of protective immunity, had also offered a clue to develop vaccine by selecting specific epitopes to make it effective. The $S$ protein and mRNA are the novel vaccine preparations. Another problem is use of effective adjuvant, as elderly people are highly susceptible to the virus and poor responders to vaccination. IgM antibodies detectable in 7-10 days after disease onset and seroconversion developed in most recovered patients.

The following factors impede the thorough immunological studies:

- How asymptomatic individuals escalate the effective humoral and cellular immune response?

- How the lymphocytes get decreased including their abnormal functionality? More knowledge is required to understand disease progression.

- Impaired regulatory mechanism of cells and cytokines favoring immune interruption for virus to propagate fast.

More attention is required in this crucial challenge of deteriorated immunological interactions to address the safe and effective therapeutics to recover the systemic interactions. 
Children develop mild response to COVID-19, because their immune system is still developing and they have the high plasticity in their adaptive immunity. Children vaccines should be developed with adjuvants to administer in early life [34].

Women are shown to have some differences in developed immune response against SARS CoV-2, as compared to men, such as:

- Less viral load and inflammation with higher CD4+ T-cells and antibody responses.

- Lower expression of serine proteaseTMPRSS2 (S-protein priming) in tissues and ACE2 in lungs.

- Overexpressed TLR8, CD40L, and CXCR3 (encoded in Xchromosome) influence the antiviral response.

- Overexpressed TLR7(a sensor for RNA viruses like Corona) is triggered by virus leads to IFN-1 and lower IL-6 production.

The lower frequency of infection and mortality in women could be related to hormonal and genetic differences, incidence of comorbidities, higher prevalence of autoimmune and allergic disorders decrease their regulatory mechanism $[10,19,26]$.

In Wuhan, the hospitalized cases of asthma didn't represent the high-risk factor for SARSCoV-2 morbidity and mortality. The relationship between the two is recommended to be studied in future $[26,41]$. Most notable features were:

- Atopic status is already related to the impairment of immune regulatory mechanism could favor antiviral immunity.

- Higher proportion of eosinophils, the immune effector cells play a protective role

- Nebulized therapeutics for topical applications in asthma by nasal airways can speed up the recovery.

- Epithelial cells of mucosa of patients with allergy express less ACE-2 and IL-13.

Impaired immune response with pathogen escaping the protective immunity

One more theory is that pathogen escape/avoid the immune system as to evade the detection by restraining the human immune responses. Susceptible HLA types, high viral loads and previously impaired immunity may contribute to the viral escape of protective immune response based on the previous knowledge obtained on other human RNA corona viruses. IFN-1 recognition and signeling is stopped by infected cells and suppressed through different processes interfering with RNA receptors. This will raise the concern that how much IFN-1 is reduced to lower down the immune response leading to the severe consequences, could be a better value for further therapy. Some intensive care units in Italy included inhaled IFN-1 $\beta$ in therapeutic protocols $[13,26]$.

TLR ligands induce the unwanted polarization towards inefficient and incompetent response in relation to innate and adaptive immunity. It compromises of the NK cells, and mediate the editing of dendritic cells and macrophages activity [24]. As a result, no TH1- nor TC1- mediated efficient antivirus response could be elicited. Hence, $80 \%$ lymphopenia is noticed especially in ICU patients, corresponding to the disease severity. The autopsy samples of lung infiltrates consist of activated macrophages with minimal lymphocytic component [26]. More studies are required to be carried out to get the information to strengthen this cytopathic pathogenesis guiding lymphopenia.

Adaptive immune process is another big concern as MHC class I and II presents virus/antigen may be compromised with antigen presenting cells, leading to impaired T-cell response [33]. The viral mutations and its possible superantigen components, leading to chronic stimulation with exhaustion of T-cell response may favor the T-cell suppression and can deviate to less protective cell profiles i.e. TH2. Detection of cytokines during mild lymphopenia could be informative to the prognosis [11]. Conserved immunodominant T-cells epitopes and their identification shall have implications for vaccine design [29].

ACE-2 (angiotensin-converting enzymes-2) receptors allow the spike protein of virus to attach and invade the cells. However, cellular proteaseTMPRSS2 are also required for virus entry. ACE-2 largely distributed to the alveolar cells, especially AT2 which also express TMPRSS2 protease [19]. On the other hand, bone marrow, lymph nodes, thymus, spleen, immune cells T/B lymphocytes and macrophages are always ACE- 2 negative. These findings suggest that immunoglobulin therapy can help treat patients with virus infection. The "Cytokine storm", is a current trademark for SARS CoV-2 pathogenesis, is triggered by releasing cytokines GSCF, IP10, MCP1, MIP1A, IL-2, IL-6, IL-7 and TNF causing pulmonary edema, problems in air exchange, ARDS, acute cardiac injury etc. leading 
the death. Vitamin B3 is also highly effective in preventing lung tissue damage.

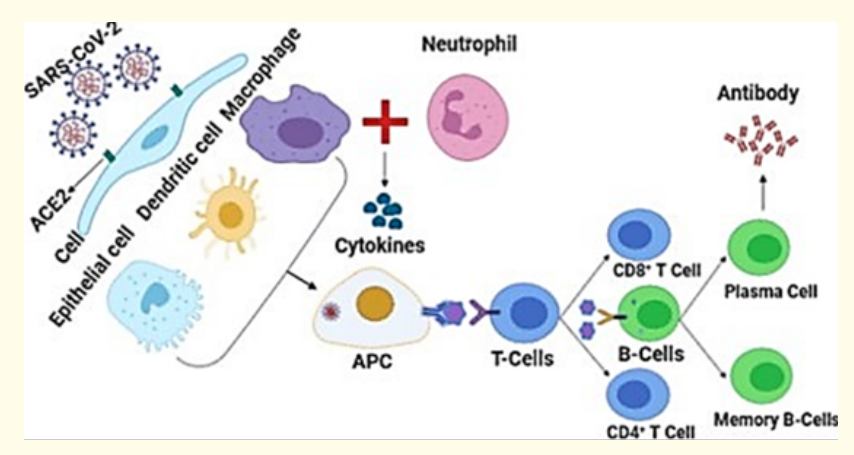

Figure 1: SARSCoV-2 entry through ACE-2 receptors activates neutrophils and macrophage to produce cytokines. MHC-Antigens on APC help to reduce the T cells activation.

One more factor in the immune evasion/lapse at different viral load is the pre-existing impaired immune response may also contribute to the immunopathogenesis. In this situation, the hyperinflammatory immune response is established due to activated persistent monocytes and macrophages.

Viral RNAs are essentially recognized by TLRs (7/8,3), to activate 3 intracellular pathways (MyD88 and IRF7) to produce IFN-1, for T-cell activation (through TRAF6 and IRF3) and production of proinflammatory molecules (through p38MAPK and AP-1). It has been hypothesized that corona RNA viruses inhibit the first two pathways leading to lower the protective immune response, but they do intensify the activation of p38MAPK expression pathway in monocytes and macrophages to exaggerate the transcription and biosynthesis of cytokines, chemokines, and acute phase proteins. Proinflammatory condition or macrophage activation syndrome is recognized in IC patients with high level of cytokines (IL-1 $\beta$, IL-6, $\mathrm{TNF} \alpha$ ) and chemokines (CCL2, CCL3, CCL5, CXCL8, CXCL10) indicate the disease progression with massive immune cells deposition in tissues $[10,30]$. In case of patients with developed pneumonia, the hypersensitive situation turns into ARDS. Others can develop secondary hemophagocytic-lymphohistiocytosis (HLH) with hypercytokinemia with multiorgan failure leading to death, reported in some COVID-19 cases [27].
Some patients have also shown to develop the disseminated intravascular coagulations due to cytokine driven epithelial and endothelial cell damage/apoptosis with increase vascular leakage, hyperexpression of tissue factors, and chronic triggering of coagulation. Comorbidities and concomitant medications (ACE inhibitors) can influence/enhance the cascade of events $[25,26]$.

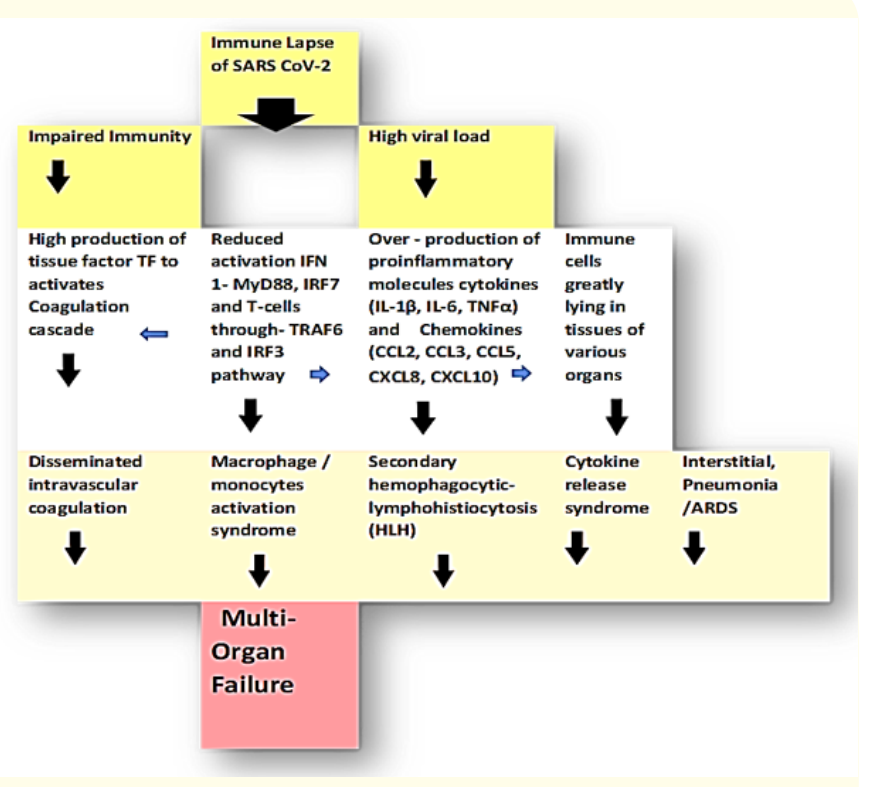

Figure 2: SARSCoV-2 lapse/evade the protective immune pathways and increasing the inflammatory activities.

Over production of cytokines to develop the inflammatory response in respiratory tract of COVID-19 patient

A metatranscriptomic sequencing is used to profile immune signatures in the bronchoalveolar lavage fluid of eight COVID-19 cases in a study. Expression of the proinflammatory genes, especially chemokines, was markedly elevated in COVID-19 cases compared to community -acquired pneumonia patients and healthy controls, suggesting that SARS CoV-2 infection cause hypercytokinemia. SARSCoV is thought to induce IFN response (IFN), but in SARSCoV-2 consistently trigger the expression of numerous IFNstimulated genes (ISGs). These ISGs genes exhibit immunopathogenic potential, and the transcriptomic data showed enhanced activation of dendritic cells and neutrophils.

More studies are needed to be done on the activation of dendritic and neutrophils cells and subsequent in situ host response to 
SARS CoV-2 could provide a rationale on disease pathogenesis and other antiviral strategies [40].

Many other transcriptomic profiling studies have been conducted previously by using intermittent peripheral blood samples [7].

In patients with ARDS, the alveolar spaces are occupied by the infiltered neutrophils and monocytes providing the pathogenic role of these cells leading to hypercytokinemia.

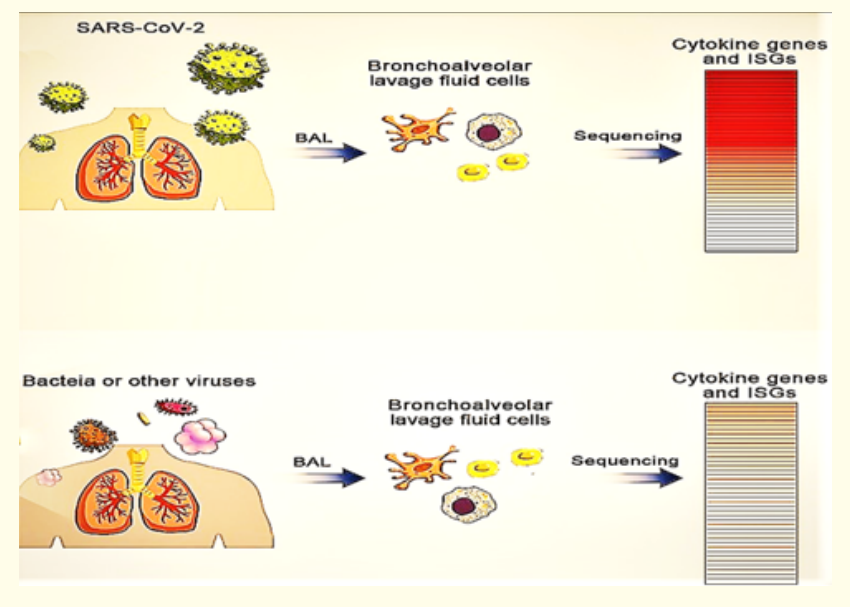

Figure 3: Cytokine and ISG gene profiling showed the higher conc. in SARSCoV-2 bronchoalveolar lavage fluid.

The SARS CoV-2 triggered a consistent IFN response traded by ISGs hyperexpression, opposite to SARS CoV. This protective potential of ISGs would be accounted for less proportion of case severities and fatalities [37]. Another exploration contrary to the former study showed that SARSCoV-2 virus can develop countermeasure against IFN system, failed to reveal the upregulation of IFN genes, by inhibiting the innate immune response [41]. These discrepancies could be addressed/investigated for justification in future.

In SARS CoV, the delayed IFN $\beta$ treatment failed to inhibit virus replication, pulmonary inflammation and aggravation [6]. The pro inflammatory ISGs can assist the antiviral protection by amplifying inflammatory signals to the body environment. IFN treatment could be/has been adopted as antiviral therapy against SARS CoV-
2 infection if the timing and dose should carefully be considered.

Mesenchymal cell therapy, a possibility for endogenous repair of the damaged cells

Under severe condition of patients with COVD-19 is mainly caused by cytokine release syndrome (CRS) mediated by leucocytes mainly, but not T-cells. Patients have received CAR-T cell therapy suggesting; a high WBC count in common with lymphocytopenia; a differential criterion for COVID-19. Blocking IL-1, IL-6 (pro-inflammatory) and TNF $\alpha$ also benefit patients. Clinical trials are conducted in China to treat COVID-19 patients with Mesenchymal Stromal/stem cells (MSCs). Clinical outcomes measures as the overall survival at 30-day post intervention with clinical and radiological improvements with negative PCR and patients were discharged from hospital on $30^{\text {th }}$ day [39].

Mesenchymal cells having immunomodulatory properties to induce the natural T-cells and M2 macrophages, capable to reverse the hyper reaction of the immune system in critical cases. A patient with COVID-19 (critical ARDS case on ventilator) was treated with PLX mesenchymal cells, had recovered. This treatment was given to him under FDA's compassionate program as a part of Coronavirus Treatment Program in US (Emergency Program) for possible therapy [12].

MSCs need to be activated by IFN $\gamma$ to strengthen their immunomodulatory effect, which may be absent in severely SARS CoV-2 affected patients as T-cells are not well activated in this infection.

In a "Licensed Approach" MSCs are treated with/without TNF/ or IL-1 could be more effective to suppress the hyperactive immune response and to promote tissue repair [28,35].

Number of potential trials are being carried out; despite the limited evidence in support of MSCs therapy, could be a revolution to treat COVID-19 patients. It is imperative that the production of MSCs are carried out to comply with strict GMP regulations.

MSC therapy can prevent the inflammatory storm related to the increased production of cytokines by immune system and promote endogenous repair by reparative properties of the stem cells [27].

Interleukin 17-A involves to promote prothrombic state in the vascular system 
Interleukin-17A (IL-17A), is a key molecule of innate and adaptive immunity. it could potentially promote the pro-thrombotic state in the vascular system. It supports the aggregate formation at sites of vascular injury [15].

The prognosis of COVID-19 is also associated with clinical risks with concomitance of cardiovascular disease, hypertension and diabetes mellitus. Cardiovascular manifestations are commonly complicated with coagulopathy related to intravascular coagulation (DIC) and/thrombic/and thromboembolic disease. Thromboembolic disease is the direct consequence of the systemic inflammatory process related to IL-6 and IL-17A $[4,5]$.

This clinical scenario is prompted the use of therapy with intravenous immunoglobulin (IVIG) and low mole. weight heparin (LMWH) anticoagulant particularly when there is a decrease in circulating T/B cells, and abnormal increase in inflammatory cytokines and D-Dimer. IVIG and LMWH have shown efficacy to treat influenza and SARS patients [1].

Based on these hypotheses, the characterization of IL-17 level is required to explore in Bronchoalveolar lavage fluid (BALF), plasma, and serum. Raising antibodies against IL-17A alone or along with in a sequential therapy with anti- IL-6, could be a therapeutic strategy to treat the cardiovascular manifestations.

Major Histocompatibility - complex Antigens/HLA alleles augmentation in SARSCoV-2 infection

Major Histocompatibility-complex antigen loci (HLA alleles) contribute to the susceptibility towards pathogenic diseases. Lymphocytes with CD4+ or CD8+ T-cells recognize the conformational structure of antigen binding slot with associated peptide. Hence, HLA haplotypes (Class I, II, and III) are associated with distinct disease susceptibilities. HLA haplotypes determine the survival during evolution. It would be advantageous if HLA molecule show special binding with SARS CoV-2 peptides on the surface of antigen presenting cells. Murine HLA class II is associated with H1N1, in human HLA class I is associated to influenza A(H1N1) pdm09 infection. Hence, it is important to identify the alleles of HLA associated with SARS CoV-2, to demonstrate the induction of [14] protective immunity. Strategic information of HLA typing/dominant alleles is necessitated to:

- Explore respective alleles and develop tests kits.

- Assist in clinical management.
- Evaluate the vaccines outcome in individuals with various susceptibility level.

- Provide susceptibility information to build strategy in the management of prevention, treatment, vaccination and clinical approaches

\section{A potential cause of fatality - hyaluronan}

The innate immune response against the virus could lead to ARDS with lungs failure and subsequent fatality. ARDS symptoms are as short/rapid breathing and cyanosis require ventilators to support in breathing. CT scan of lungs showed grounded glass patches containing jelly like liquid fluid, hyaluronan (HA) is associated with ARDS [36,38]. The inflammatory cytokines (IL-1 and TNF) are high in number induce HA-synthase (HAS2) in CD31- endothelium, EpCAM- lung alveolar epithelial cells, and fibroblasts [2]. HA absorb water in significantly higher amount, 1000 time as of its mole. wt. Hence, inhibiting the HA production do support patient in breath, which holds great promise. Doctors can use a medical grade hyaluronidase enzyme to reduce accumulation of HA and Hymecromone (4-Methylumbelliferone,4-MU) to inhibit HAS2.

Two phased immune response take place in lungs. The first immune defense is the protective phase and the second inflammationdriven is damaging phase. Immune response should be boosted in the first phase, while suppressing the second phase. Vitamin B3 is highly lung protective, it should be used as coughing begins. At the

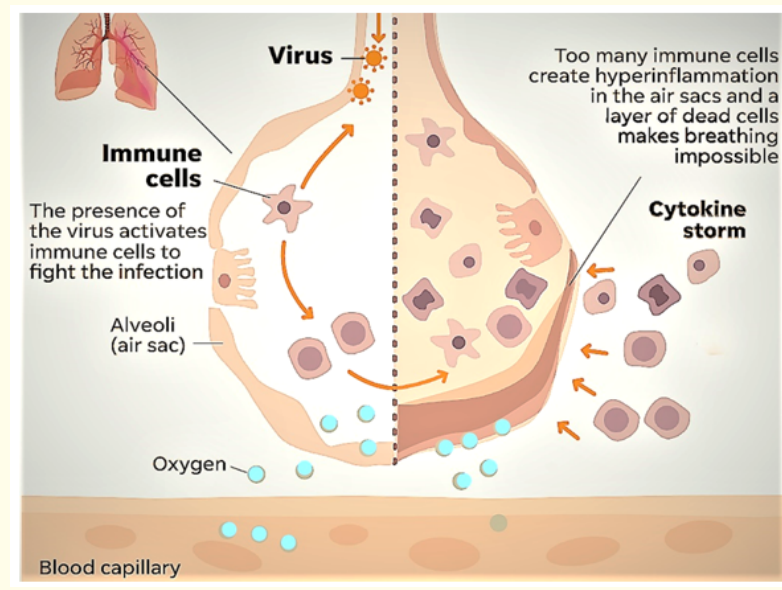

Figure 4: Schematic representation of two phased immune response in lungs: Protective (Left) and Inflammatory (Right). 
time of difficulty in breathing the treatment with hyaluronidase is used intratracheally and 4-MU is given to inhibit HAS2.

\section{Discussion}

Innate immune response and pathogenesis is enhanced in SARSCoV-2, basically in three states:

- Immunosuppressive state.

- Normal immune state

- Hyperimmune state.

High leukocyte numbers, C-reactive protein (CRP), erythrocyte sedimentation rate, D-dimer and an imbalanced pro/and antiinflammatory cytokines and chemokines with higher expression levels of IL-2, IL-7, IL-10, G-CSF, IP-10, MCP-1, MIP1- $\alpha$, and TNF- $\alpha$ were found in individuals admitted to the intensive care unit (ICU) [18]. These cytokines indicate that patient is at risk to develop lung injury, subsequently ARDS which mostly have fatal consequences. This situation is denoted as cytokine release syndrome (CRS) or "cytokine storm" [27].

SARSCoV-2 is first attached with ACE-2 receptors in nasopharynx and respiratory epithelium [19]. Host immune response is initiated as pro-inflammatory cytokines are released from various lymphocytes [16]. Pro-inflammatory cytokines are induced to determine the lung injury, turning this into more severe form ARDS, which could be associated to high mortality. This effect is more induced and is articulated more in older age or comorbid situation as they procure more ACE- 2 receptors. Comorbid situations of patients may have pre-existing subclinical inflammations like arteries inflammations, represented by increased highly sensitive CRP (hsCRP) in these groups. . It has a lesser effect in younger age groups, because of less developed ACE-2 receptors, immature immune system and the predominance of Th2 immunity than Th1. Hyper activation of various immune cells especially against N-protein of COVID-19, help forming the "cytokine storm" resulting to cause acute lung injury, ARDS followed by multiorgan failure.

There is a high degree non-correlation in clinico-pathology. Patients can be asymptomatic/with mild conditions, despite the severely progressed conditions, which will delay the appropriate treatment at an early stage. There is more immunological activation than the other usual respiratory viruses.
Evidence based medicines integrates the clinical experience and patient values with the best available research information [20]. Many clinicians are applying treatment to current COVID-19 cases based on the previous experience with MERS and SARS. However, it is difficult to find appropriate new drug for COVID-19 amid pan-

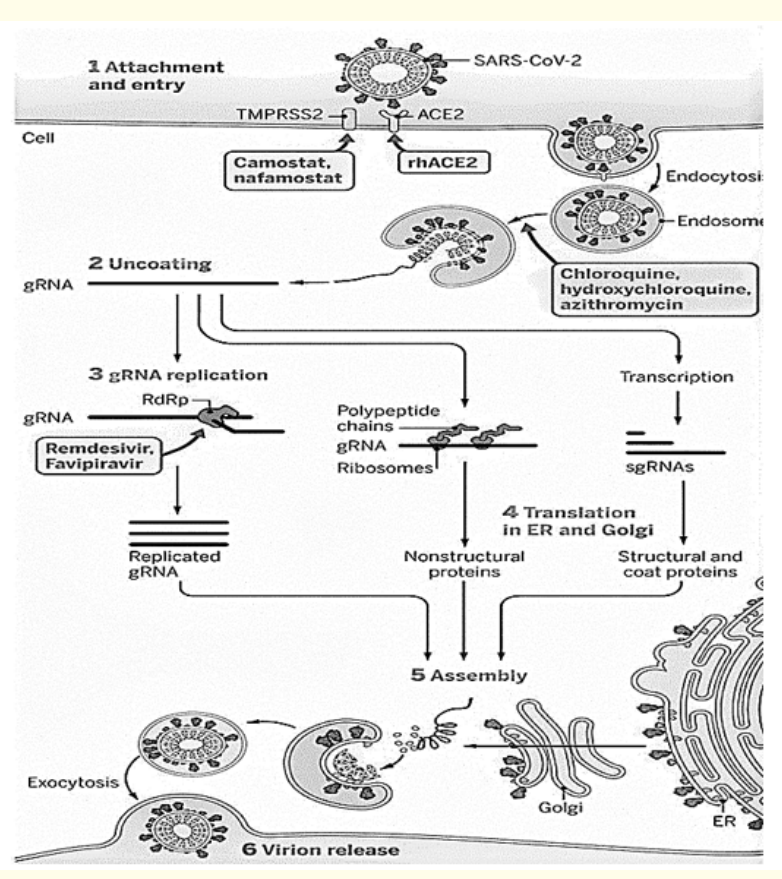

Figure 5: Possible targets for drugs to stop propagation cycle of virus at various stages.

demic, because it takes long time to develop medicines. Concomitant efforts have been done during pandemic for COVID-19 treatment as for various drugs.

It is critical to complete medical studies to determine whether any of these medications are effective against COVID-19. Some of the following drugs, the researchers are studying/and trialing to control COVID-19.

- Dexamethasone/corticosteroid is an anti-inflammatory, reduce the death by $30 \%$ for people on ventilators. It inhibits the expression of various genes encoding inflammatory molecules especially offset the potential "cytokine storm".

- Lopinavir and ritonavir are anti-HIV protease inhibitors. 
They also work against Coronaviruses to inhibit 3-chymotripsin-like protease. It reduced the symptoms elevation in groups with lopinavir, ritonavir, IFN $\beta$ and ribavirin, as compared to a group receiving lopinavir and ritonavir alone.

- Tocillizumab and sarilumab are the monoclonals against IL-6 cytokines reduce the cytokine release syndrome.

- Favipiravir inhibit the RNA polymerase.

- Remdesivir is a new prodrug of nucleoside GS-441524 which stops the virus replication.

- Chloroquine and Hydroxychloroquine aims to target the virus replication and blocks the glycosylation of host receptors to breakdown the protein production. This medicine is turned down by FDA recently, as it increases the heart rhythm problem.

- Nefamostat and chemostat are, approved in Japan, both acting as antagonistic to serine protease TMPRSS2, block the entry of SARSCoV-2 in-vitro. They are on 2nd -3rd trial in US to treat COVID-19 patients.

- Famotidine is thought to bind papain like protease encoded by SARSCoV-2, which help in virus entry to the host cells. Its trials are going on with hydroxychloroquine.

- Umifenovir is an indol derivative hydrophobic molecule interact with lipids and proteins affecting the cellular trafficking of the virus.

- Nitazoxanide blocks the maturation of viral N-nucleocapsid protein, promotes the reduction of viral particles.

- Ivermectin destabilizes the cell transport proteins used to enter nucleus.

- Bevacizumab reduces the vascular permeability to decrease the fluid entering the lungs.

- Fluvoxamine is immunomodulator as serotonin-reuptake inhibitor binds to sigma-1 receptor to shut down the inflammatory cascade from endoplasmic reticulum.

All of these drugs are under repurposing trial studies under scrutiny [22].

\section{Conclusion}

COVID-19 patients are divided into two categories: One is person with virus positive results are isolated at home and other one, when person is detected with high viral load with severe symp- toms and admitted to the hospital. Some patients with moderate symptoms treated with steroids and antibiotics. If they progress to severe condition, they will be admitted in Hospital to get the appropriate treatment. Policies are made that medication should be given to patients in reasonable and humanitarian way than the usual situation.

Efforts should be made to detect asymptomatic/or people with mild symptom as they can develop severe symptoms with bacterial complications in lungs, should start with early treatment.

Efforts should made to develop diagnostics for early detection, hence preventing the process establishing "Cytokine storm" situation.

Randomized Clinical Trials (RCT) are meaningful, often reversed when larger trial is performed. Meta analytic results are the best evidence, should be interpreted with cautions, but they can be significant even if the results of individual RCTs have no statistical significance [9].

It is important that in the pandemic era, publications should be/are to be published urgently even though they are case reports, case series and small communication. It will assist scientific community to share the essential knowledge

We should also provide elderberry supplements and betadine mouth or nasal spray which are not causing significant harm [17].

There is less information available on the ACE-2 and their blockers ARBs, and it is a physio pathological mechanism, but the substantial benefits/risks evaluation with ACE and ARB can't be recommended. Blocking entry of virus could be a potential therapeutic target.

Misinterpretation of studies are causing significant harm [3]. The role of experts who are doing evidence-based medicine or meta-research is very important to reduce harm amid pandemic.

\section{Acknowledgements}

The author has extended her gratitude and obligation towards Dr. Rajesh K Gupta, President and Principal Consultant at Biologics Quality and Regulatory Consultants, Washington D.C. Metro Area; for his valuable guidance and support. 


\section{Bibliography}

1. Atri D., et al. "COVID-19 for cardiologist: a current review of the virology, clinical epidemiology, cardiac and other clinical manifestations and potential therapeutic strategies". ACC: Basic to Translational Science (2020).

2. Bell TJ., et al. "Defective lung function following influenza virus is due to prolonged, reversible hyaluronan synthesis". Matrix Biology 80 (2018): 14-28.

3. Bero L., et al. "The SSSPIN study-spin in studies of spin: meta research analysis”. BMJ 367 (2019): 16202.

4. Casillo GM., et al. " Could IL-17 represent a therapeutic target for the treatment and/or management of COVID-19 related respiratory syndrome? This paper is dedicated to Sofia Maione born during COVID-19 outbreak". Pharmacology Research (2020).

5. Caso F., et al. "Could Sars-coronavirus-2 trigger autoimmune and / or autoinflammatory mechanisms in genetically predisposed subjects?" Autoimmun Review 19.5 (2020): 102524.

6. Channappanavar R., et al. "IFN-I response timing relative to virus replication determines MERS coronavirus infection outcomes". Journal of Clinical Investigation 130 (2019): 36253693.

7. Chaussabel D. "Assessment of immune status using blood transcriptomics and potential implications for good health". Seminar in Immunology 27 (2015): 58-66.

8. Chen X., et al. "Human monoclonal antibodies block the binding of SARS-CoV-2 spike protein to angiotensin converting enzyme 2 receptor". Cellular and Molecular Immunology 17 (2020): 647-649.

9. Choi EK., et al. "Body mass index and 20 specific cancers : re- analysis of dose- response meta-analysis of observational studies". Annuals in Oncology 29 (2018): 749-757.

10. Conti P., et al. "Coronavirus COV-19/ SARS -COV-2 affects women less than men: clinical response to viral infection". Journal of Biological Regulators and Homeostatic Agents 34.2 (2020).

11. Cossarizza S., et al. "SARS-CoV-2, the virus that cause COVID-19: cytometery and the new challenge for global health". Cytometry 97 A (2020): 340-343.
12. Dana Rubin. "Pluristem expands its compassionate Use Program: Treated first COVID-19 patient in US. Under FDA Single Pateint expanded access program".

13. De Wit E., et al. "SARS and Mers: recent insights into emerging coronaviruses". Nature Reviews Microbiology 14 (2016): 523534.

14. Dutta M., et al. "Polymorphism of HLA class I and class II alleles in influenza A (H1N1) pdm 09 virus infected population of Assam, Northeast India". Journal of Medical Virology 90 (2018): 854-860.

15. Federica Raucci., et al. "Interleukin-17 A (IL-17A), a key molecule of innate and adaptive immunity, and its potential involvement in COVID-19 related thrombotic and vascular mechanisms". Autoimmunity Reviews 19.7 (2020).

16. Fu Y., et al. "SARS-CoV-2 mediated inflammatory response: from mechanism to potential therapeutic tools". Virologica Sinica 35 (2020): 266-271.

17. Hawkins J., et al. "Black elderberry (Sambucus nigra) supplementation effectively treat upper respiratory symptoms: a meta analysis of randomized controlled clinical trials". Complementary Therapies in Medicine 42 (2019) 361-365.

18. Huang C., et al. "Clinical features of patients infected with 2019 novel coronavirus in Wuhan, China". Lancet 395.10223 (2020): 497-506.

19. Hoffmann M., et al. "SARS-CoV-2 cell entry depends on ACE-2 and TMPRSS2 and is blocked by a clinically proven protease inhibitor". Cell 181 (2020): 271-280.

20. Izet Masic., et al. "Evidence based medicine-new approaches and challenges". Acta Informatica Medica 16.4 (2008): 219-225.

21. Kikkert M., et al. "Innate immune evasion by human respiratory RNA viruses". Journal of Innate Immunity 12 (2020): 4-20.

22. Leah Shaffer. "15 drugs being tested to treat COVID-19 and how they would work". Nature (2020).

23. LI G., et al. "Coronavirus infections and immune responses". Journal of Medical Virology 92.4 (2020): 424-432.

Citation: Anju Kaushal. "Immune Response and Pathogenesis of COVID-19 and The Strategies for Developing Target Drugs". Acta Scientific Microbiology 3.9 (2020):: 92-102 
24. Locati M., et al. "Diversity, mechanisms and significance of macrophage plasticity". Annual Review of Pathology 15 (2020): 123-147.

25. Liao M., et al. "The landscape of lung bronchoalveolar immune cells in COVID-19 revealed by single-cell RNA sequencing". medRxiv (202).

26. Maggi E., et al. "COVID-19 Unanswered questions on immune response and pathogenesis". Journal of Allergy and Clinical Immunology 146 (2020): 18-22.

27. Mehta P., et al. "COVID-19: consider cytokine storm syndromes and immunosuppression". Lancet 395 (2020): 1033-1034.

28. Metcalfe SM. "Mesenchymal stem cells and management of COVID-19 pneumonia". Medicine in Drug Discovery (2020).

29. Peter B., et al. "T-cell epitope predictions. T cell epitope predictions". Annual Reviews in Immunology 38 (2020): 123-145.

30. Perlman S., et al. "Immunopathogenesis of coronavirus infections: implications for SARS". Nature Review Immunology 5 (2005): 917-927.

31. Rothe C., et al. "Transmission of 2019-nCoV infection from an Asymptomatic Contact in Germany". The New England Journal of Medicine 382 (2020).

32. Shi Y., et al. "COVID-19 infection: the perspectives on immune response”. Cell Death and Differentiation 27 (2020): 14511454.

33. Shin HS., et al. "Immune response to middle east respiratory syndrome coronavirus during the acute and convalescent phase of human infection". Clinical Infectious Diseases 68 (2019): 984-992.

34. Wang A., et al. "Single nucleus multiomic profiling reveals agedynamic regulation of host genes associated with SARS-CoV-2 infection". BiorXiv (2020).

35. Wang G., et al. "Kynurenic acid, an IDO metabolite, control TSG6-mediated immunosuppression of human mesenchymal stem cells". Cell Death and Differentiation 25 (2018): 1209-1223.

36. Wang D., et al. "Clinical Characteristics of 138 Hospitalized patients with 2019 Novel Coronavirus-Infected Pneumonia in Wuhan, China". JAMA (2020).
37. Wu Z., et al. "Characteristics of and important lessons from the coronavirus disease 2019 (COVID-19) Outbreak in China: Summary of a Report of 72314 cases from the Chinese Centre for Disease Control and Prevention". JAMA (2020).

38. Xu Z., et al. "Pathological findings of COVID-19 associated with acute respiratory distress syndrome". The Lancet Respiratory Medicine (2020).

39. Zaineb Akram Dr. "Mesenchymal Stem Cell Infusion for COVID-19 Infection". National Institute of Blood and Marrow Transplant (NIBMT), Pakistan. NIH.US National Library of Medicine.

40. Zhau Zhau., et al. "Heightened immune responses in the respiratory tract of COVID-19 patients". Cell Host and Microbe 32.6 (2020): 883-890.e2

41. Zhou F., et al. "Clinical course and risk factors for mortality of adult patients with COVID-19 in Wuhan, China: a retrospective cohort study". Lancet 395 (2020): 1054-1062.

\section{Assets from publication with us}

- Prompt Acknowledgement after receiving the article

- Thorough Double blinded peer review

- Rapid Publication

- Issue of Publication Certificate

- High visibility of your Published work

Website: www.actascientific.com/

Submit Article: www.actascientific.com/submission.php

Email us: editor@actascientific.com

Contact us: +919182824667

Citation: Anju Kaushal. "Immune Response and Pathogenesis of COVID-19 and The Strategies for Developing Target Drugs". Acta Scientific Microbiology 3.9 (2020):: 92-102 